\title{
The Effects of Monosodium Glutamate on PSMA Radiotracer Uptake in Men with Recurrent Prostate Cancer: A Prospective, Randomized, Double-Blind, Placebo-Controlled Intraindividual Imaging Study
}

\author{
Sara Harsini ${ }^{1}$, Heather Saprunoff ${ }^{1}$, Tina Alden ${ }^{1}$, Behnoud Mohammadi $^{1}$, Don Wilson ${ }^{1}$, and François Bénard ${ }^{1,2}$ \\ ${ }^{1}$ BC Cancer, Vancouver, British Columbia, Canada; and ${ }^{2}$ Department of Radiology, University of British Columbia, Vancouver, \\ British Columbia, Canada
}

The prostate-specific membrane antigen (PSMA) is an excellent target for theranostic applications in prostate cancer. However, PSMA-targeted radioligand therapy can cause undesirable effects due to high accumulation of PSMA radiotracers in salivary glands and kidneys. This study assessed orally administered monosodium glutamate (MSG) as a potential means of reducing kidney and salivary gland radiation exposure using a PSMA-targeting radiotracer. Methods: This prospective, double-blind, placebo-controlled study enrolled 10 patients with biochemically recurrent prostate cancer. Each subject served as his own control. PET/CT imaging sessions using 2-(3\{1-carboxy-5-[(6-18 F-fluoro-pyridine-3-carbonyl)-amino]-pentyl\}-ureido)pentanedioic acid ( $\left.{ }^{18} \mathrm{~F}-\mathrm{DCFPyL}\right)$ were performed 3-7 d apart, after oral administration of either $12.7 \mathrm{~g}$ of MSG or placebo. Data from the 2 sets of images were analyzed by placing regions of interest on lacrimal, parotid, and submandibular glands; left ventricle; liver; spleen; kidneys; bowel; urinary bladder; gluteus muscle; and malignant lesions. The results from MSG and placebo scans were compared by paired analysis of the region-of-interest data. Results: In total, 142 pathologic lesions along with normal tissues were analyzed. As hypothesized a priori, there was a significant decrease in SUV $V_{\max }$ corrected for lean body mass $\left(S U L_{\max }\right)$ on images obtained after MSG administration in the parotids $(24 \% \pm 14 \%, P=0.001)$, submandibular glands $(35 \% \pm 11 \%, P<0.001)$, and kidneys $(23 \% \pm$ $26 \%, P=0.014)$. Significant decreases were also observed in the lacrimal glands $(49 \% \pm 13 \%, P<0.001)$, liver $(15 \% \pm 6 \%, P<0.001)$, spleen $(28 \% \pm 13 \%, P=0.001)$, and bowel $(44 \% \pm 13 \%, P<0.001)$. A mildly lower blood pool SUL mean was observed after MSG administration (decrease of $11 \% \pm 13 \%, P=0.021$ ). However, significantly lower radiotracer uptake in terms of SUL mean, $S U L_{\text {peak }}$, and $S U L_{\text {max }}$ was observed in malignant lesions on scans performed after MSG administration than on the placebo studies (SUL $L_{\max }$ median decrease, $33 \%$; range, $-1 \%$ to $75 \%$; $P<0.001$ ). No significant adverse events occurred after placebo or MSG administration, and vital signs were stable. Conclusion: Orally administered MSG significantly decreased salivary gland, kidney, and other normal-organ PSMA radiotracer uptake in human subjects, using ${ }^{18} \mathrm{~F}-\mathrm{DCFPyL}$ as an exemplar. However, MSG caused a corresponding reduction in tumor uptake, which may limit the benefits of this approach for diagnostic and therapeutic applications.

Received Apr. 9, 2020; revision accepted Apr. 13, 2020.

For correspondence or reprints contact: Francois Bénard, British Columbia Cancer Research Center, Room 4.113, 675 W. 10th Ave., Vancouver, BC, Canada V5Z 1 L3.

E-mail: fbenard@bccrc.ca

Published online May 8, 2020

COPYRIGHT (C) 2021 by the Society of Nuclear Medicine and Molecular Imaging.
Key Words: monosodium glutamate; PSMA; prostate-specific membrane antigen; salivary glands; kidney

J Nucl Med 2021; 62:81-87

DOI: 10.2967/jnumed.120.246983

$\mathbf{P}$ rostate cancer ( $\mathrm{PCa}$ ) is the principal cause of cancer mortality in men worldwide because of the development of metastatic disease (1). Advanced stages of PCa initially respond to androgen deprivation therapy, but within an interval of 1-3 y, they invariably develop androgen independence (2). Other therapeutic agents approved for the treatment of metastatic castration-resistant PCa (cabazitaxel (3), abiraterone (4), sipuleucel-T (5), enzalutamide (6), and ${ }^{223} \mathrm{Ra}(7)$ ) can improve survival. However, none culminated in durable clinical responses, with a survival benefit of generally less than 6 mo (6). The prostate-specific membrane antigen (PSMA), a type II carboxypeptidase-associated transmembrane glycoprotein with folate hydrolase activity, is overexpressed in PCa cells (8). Several radiolabeled PSMA ligands, showing high sensitivity or specificity for PSMA-expressing tissues, have been investigated in PET imaging (9). Current evidence suggests that PSMA-targeting radioligand therapy (RLT) shows promise to treat metastatic castration-resistant PCa patients, by using ligands such as $\beta$-emitting $\left({ }^{177} \mathrm{Lu}\right)$ or $\alpha$-emitting isotopes $\left({ }^{225} \mathrm{Ac}\right)(10,11)$.

One of the major disadvantages of PSMA-RLT is the high accumulation of the radiolabeled tracers in nontarget organs, including the salivary glands and kidneys. High accumulation of the PSMA radiopharmaceuticals in salivary glands can result in transient or permanent xerostomia, an adverse event with a variable reported rate of $8 \%-87 \%$, which is particularly problematic with ${ }^{225} \mathrm{Ac}$, leading to treatment discontinuation in many cases $(10,12)$. On the other hand, renal accumulation of $\beta$-emitters such as ${ }^{177} \mathrm{Lu}-$ PSMA-617 represents the cumulative dose-limiting toxicity and leads to risk of nephrotoxicity (13). Furthermore, the increased risk of chronic renal disease prevents initiation of PSMA-RLT earlier in the disease course (14). Many attempts-including sialendoscopy with dilatation, saline irrigation, steroid injection, intraparenchymal injections of botulinum toxin, external cooling of the salivary glands with ice packs, and oral administration of folic polyglutamate tablets-have been tried to mitigate salivary gland toxicity, with some, but limited, success (15-18). Mannitol infusion is a strategy 
to reduce renal uptake of PSMA-targeted tracers by acting as an osmotic diuretic, decreasing renal reabsorption. However, its effect on tumor uptake needs to be clarified (19). The administration of 2(phosphonomethyl)pentane-1,5-dioic acid (2-PMPA), a phosphonatebased PSMA inhibitor, reduced accumulation of PSMA radiotracers in the kidneys in a dose-dependent manner, but this reduction was generally accompanied by decreased tumor uptake (20-22). A novel 2-PMPA prodrug (e.g., tris-POC-2-PMPA) has been proposed to specifically shield the kidneys and salivary glands from PSMA-RLT; however, its effect on tumor uptake requires further studies (23). We recently reported that the administration of monosodium glutamate (MSG), a well-known food additive, reduced salivary and kidney ${ }^{68} \mathrm{Ga}$-PSMA-11 uptake in LNCap tumor-bearing mice while leaving tumor uptake unaffected (24).

In this study, we explored the effects of MSG in human subjects on the biodistribution of a PSMA-targeting radiopharmaceutical. We performed an intraindividual comparison of the biodistribution of 2-(3-\{1-carboxy-5-[(6- ${ }^{18}$ F-fluoro-pyridine-3-carbonyl)-amino]pentyl $\}$-ureido)-pentanedioic acid $\left({ }^{18} \mathrm{~F}-\mathrm{DCFPyL}\right)$ in patients with biochemical recurrence of $\mathrm{PCa}$, comparing scans performed with the prior administration of orally administered MSG or placebo. We evaluated the uptake of ${ }^{18} \mathrm{~F}-\mathrm{DCFPyL}$ in normal organs and malignant lesions to determine whether MSG could reduce offtarget binding of PSMA-targeting radiopharmaceuticals as a potential strategy to improve the therapeutic ratio of RLT.

\section{MATERIALS AND METHODS}

\section{Patients}

The study included 10 patients with either of the following criteria: known PCa with biochemical recurrence after initial curative radiation therapy, with a prostate-specific antigen level of more than $2 \mathrm{ng} / \mathrm{mL}$ above the nadir after therapy, or known PCa with biochemical recurrence after initial curative radical prostatectomy, with a prostate-specific antigen level of more than $0.4 \mathrm{ng} / \mathrm{mL}$ and an additional measurement showing increase. Exclusion criteria were medical instability; inability to lie supine for imaging; inability to provide written consent; a body weight exceeding the safe limit of the PET/CT bed $(204.5 \mathrm{~kg})$ or an inability to fit through the PET/CT bore (70-cm diameter); an Eastern Cooperative Oncology Group status of more than 2; severe uncontrolled hypertension; a history of severe asthma; a history of intolerance to MSG; a history of severe headaches or migraines triggered by food or MSG; and use of a sodium- or salt-restricted diet due to other medical conditions. Although no treatment was discontinued before the ${ }^{18} \mathrm{~F}$ DCFPyL scan, no new treatment was initiated between the first and second ${ }^{18} \mathrm{~F}$-DCFPyL scans. The patients were randomly assigned to receive either placebo or MSG before the first PET acquisition and then crossed over to the other intervention for a repeat scan within 3-7 d. The patients, research staff acquiring the studies, and scan readers did not know whether the subjects received placebo or MSG.

The study was approved by the University of British Columbia/BC Cancer Research Ethics Board. Written informed consent was provided by all participants before inclusion in the study. The study was registered on ClinicalTrials.gov (NCT03693742).

\section{Study Procedures}

Patient demographics, relevant oncologic history, laboratory values, and tumor pathology data were recorded. Participants were followed up $24 \mathrm{~h}$ after radiotracer administration to identify adverse events.

${ }^{18} \mathrm{~F}$-DCFPyL was synthesized according to a previously published method (25). The administered activity was scaled by body weight (range, 237-474 MBq), allowing a $10 \%$ variation in target activity. After a 4 -h fast and 30 min before intravenous ${ }^{18} \mathrm{~F}-\mathrm{DCFPyL}$ injection, each participant ingested either $300 \mathrm{~mL} \pm 5 \%$ of low-sodium tomato juice (Heinz) containing $12.7 \mathrm{~g}$ of MSG or regular tomato juice (Heinz; placebo), according to a computer-generated random list that determined the sequence of the scans. Second PET/CT examinations were performed within an interval of 3-7 d.

Vital signs were recorded before administration of MSG, before ${ }^{18} \mathrm{~F}$-DCFPyL injection, between 5 and $15 \mathrm{~min}$ after ${ }^{18} \mathrm{~F}-\mathrm{DCFPyL}$ injection, and $60 \mathrm{~min}$ after radiotracer injection. Between 60 and $120 \mathrm{~min}$, participants were allowed to have a meal of their choice. After a 120-min uptake period, vital signs were monitored again, and the participants were imaged from the top of the head to the mid thigh on a Discovery PET/CT 690 scanner (GE Healthcare). A CT scan for localization and attenuation correction $(120 \mathrm{kV}$, automatic $\mathrm{mA}$ selection [30-200 mA range], and noise index of 20) was acquired. PET data were acquired immediately after the CT scan over $2-4 \mathrm{~min} / \mathrm{bed}$ position, adjusted for participant girth. All images were reconstructed identically using ordered-subset expectation maximization and pointspread function modeling.

\section{Image Interpretation}

Two nuclear medicine physicians with access to all clinical data reviewed the PET/CT images reconstructed without the time-of-flight option using a MIM workstation (MIM Maestro, version 6.9.4; MIM Software Inc.). PET, CT, and PET/CT images were reviewed in axial, coronal, and sagittal planes in 2 reading sessions. The readers did not know which intervention occurred before the scan they were interpreting (MSG or control). After visual qualitative identification of the tumoral lesions, a semiquantitative evaluation was performed on the basis of SUV adjusted for the lean body mass and reflecting a maximum single-pixel uptake value $\left(\mathrm{SUL}_{\text {max }}\right) ; \mathrm{SUL}_{\text {peak }}$, calculated using an automated computed maximal average SUL in a $1.0 \mathrm{~cm}^{3}$ spheric volume within the tumor; and $\mathrm{SUL}_{\text {mean }}$. For normal organs, regions of interest were drawn at predetermined reference sites. including the lacrimal, parotid, and submandibular glands; left ventricular blood pool; liver; spleen; kidneys; bowel; urinary bladder; and gluteus muscle. The arithmetic mean was calculated for paired organs. For all malignant lesions, the $\mathrm{SUL}_{\text {max }}, \mathrm{SUL}_{\text {mean }}$, and $\mathrm{SUL}_{\text {peak }}$ were measured using the PET Edge tool running on MIM. All regions of interest were created in 1 dataset by a masked observer and then saved and compared with the identical location in the second dataset for matched comparisons of activity.

\section{Statistical Analysis}

Descriptive values were expressed as the mean \pm SD or as the median and range if data were not normally distributed according to the Shapiro-Wilk test. The relative percentages of $\mathrm{SUL}_{\max }, \mathrm{SUL}_{\text {mean }}$, and $\mathrm{SUL}_{\text {peak }}$ change between control and MSG studies were calculated as [(MSG value - control value)/control value] $\times 100$. The independent Student $t$ test was performed to compare normal variables; otherwise, nonparametric tests, including the Wilcoxon signed-rank test for paired data, were used to compare malignant lesion $\mathrm{SUL}_{\text {mean }}$, $\mathrm{SUL}_{\max }$, and $\mathrm{SUL}_{\text {peak }}$. To adjust for multiple testing and control the false-discovery rate, the Benjamini-Hochberg method was used (26). Finally, the sample was adjusted by a weight factor, using the weightcases option in the SPSS software in malignant lesion analysis, to balance the sample in accordance with the uneven frequency of malignant lesions in different patients. The correlation of percentages of SUL $_{\max }$ change between control and MSG studies (for salivary glands and kidneys) with MSG doses was compared by Pearson correlation testing. To examine changes in vital signs over the study period, an ANOVA for repeated measures was used. Statistical analyses were conducted using SPSS Statistics (version 25.0; IBM Corp.) and R (version 3.6.0; R Foundation for Statistical Computing). A $P$ value of less than 0.05 was considered significant. 


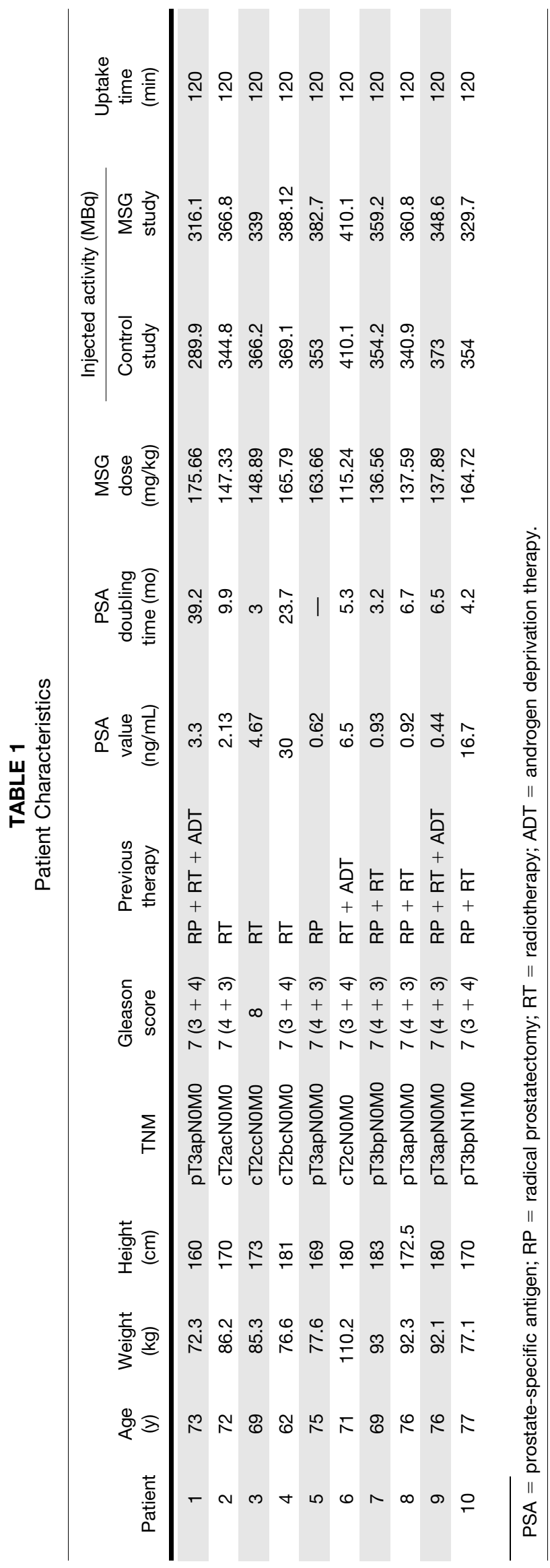

RESULTS

\section{Demographic Characteristics}

As depicted in Table 1, this prospective analysis included 10 patients (mean age, $72 \pm 4.5 \mathrm{y}$ ), of whom 6 had a biochemical recurrence after radical prostatectomy and 4 had a biochemical recurrence after curative-intent radiotherapy. Prior treatments included surgery (60\% of cases), radiotherapy $(90 \%)$, or androgen deprivation therapy (30\%), with $60 \%$ of participants having received more than 1 type of therapy. Overall, the subjects had a mean prostate-specific antigen level of $6.62 \pm 9.56 \mathrm{ng} / \mathrm{mL}$, with a doubling time of $11.3 \pm 12.2 \mathrm{mo}(n=9 \mathrm{mo})$. Representative MSG and control ${ }^{18} \mathrm{~F}-\mathrm{DCFPyL}$ PET/CT scans are shown in Figure 1.

\section{Normal Tissues}

The average $\mathrm{SUL}_{\text {mean }}$ and $\mathrm{SUL}_{\max }$ of different normal tissues in all patients for both MSG and control PET/CT are described in Table 2. A statistically significant lower $\mathrm{SUL}_{\text {mean }}$ in the MSG group than in the placebo group was noted in the left ventricle blood pool $(10.72 \% \pm 12.54 \%, P=0.03)$, liver $(15.75 \% \pm$ $7.32 \%, P<0.001)$, spleen $(34.34 \% \pm 11.11 \%, P<0.001)$, parotid glands $(26.07 \% \pm 16.98 \%, P=0.004)$, submandibular glands $(34.68 \% \pm 18.68 \%, P=0.001)$, lacrimal glands $(41.88 \% \pm$ $18.88 \%, P<0.001)$, bowel $(45.08 \% \pm 13.83 \%, P<0.001)$, and kidneys $(27.39 \% \pm 12.07 \%, P=0.001)$. The $\mathrm{SUL}_{\text {mean }}$ was lower in the gluteus muscle $(7.92 \% \pm 21.72 \%, P=0.17)$ and urinary blad$\operatorname{der}(14.85 \% \pm 30.22 \%, P=0.08)$, but this difference did not reach statistical significance.

In addition, the $\mathrm{SUL}_{\max }$ was significantly lower in the liver $(14.60 \% \pm 5.75 \%, P<0.001)$, spleen $(28.27 \% \pm 13.39 \%, P=$ $0.001)$, parotid glands $(23.98 \% \pm 14.03 \%, P=0.001)$, submandibular glands $(35.03 \% \pm 11.19 \%, P<0.001)$, lacrimal glands $(48.53 \% \pm 12.54 \%, P<0.001)$, bowel $(43.77 \% \pm 12.95 \%, P<$ $0.001)$, and kidneys $(23.46 \% \pm 26.08 \%, P=0.014)$. The lower $\mathrm{SUL}_{\max }$ in the left ventricle blood pool $(11.42 \% \pm 19.08 \%$,

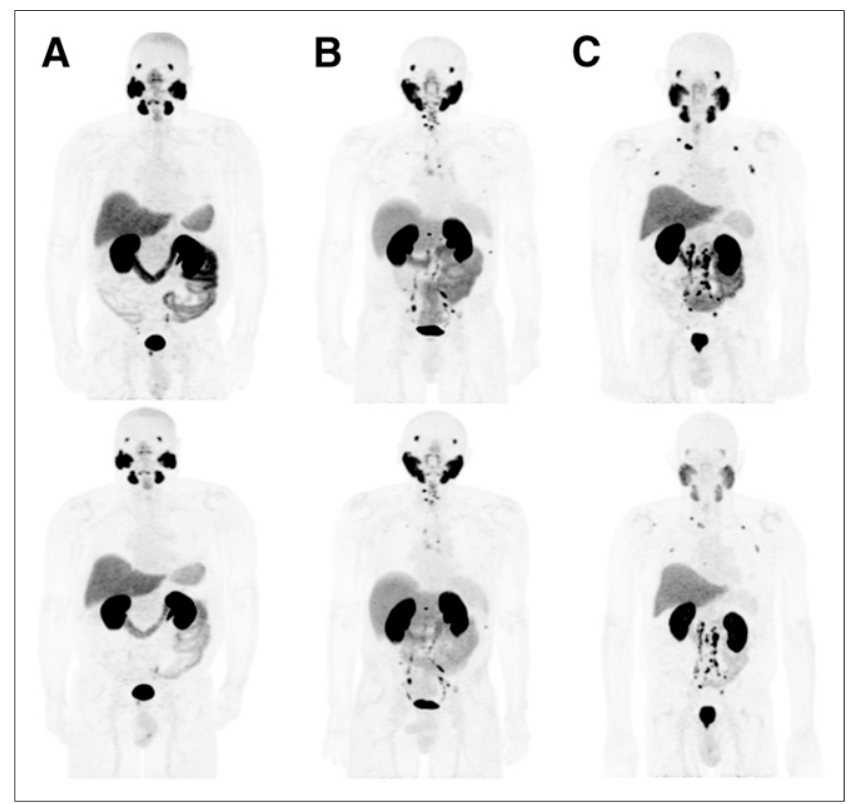

FIGURE 1. Representative anterior maximum-intensity projections of patients $2(\mathrm{~A}), 4(\mathrm{~B})$, and $10(\mathrm{C})$, with placebo images in upper row and MSG images in bottom row. 
TABLE 2

Comparison of ${ }^{18}$ F-DCFPyl Uptake in Normal Tissues in Control and MSG Studies

\begin{tabular}{|c|c|c|c|c|c|c|c|c|}
\hline \multirow[b]{2}{*}{ Tissue } & \multicolumn{4}{|c|}{ Average SULmean } & \multicolumn{4}{|c|}{ Average SUL $L_{\max }$} \\
\hline & $\begin{array}{l}\text { Control } \\
(n=10)\end{array}$ & $\begin{array}{c}\text { MSG } \\
(n=10)\end{array}$ & $\begin{array}{c}\text { Decrease } \\
(\%)(n=10)\end{array}$ & $P^{*}$ & $\begin{array}{l}\text { Control } \\
(n=10)\end{array}$ & MSG $(n=10)$ & $\begin{array}{c}\text { Decrease } \\
(\%)(n=10)\end{array}$ & $P^{*}$ \\
\hline Blood pool & $0.8 \pm 0.1$ & $0.7 \pm 0.1$ & $10.7 \pm 12.5$ & 0.021 & $1.1 \pm 0.2$ & $0.9 \pm 0.2$ & $11.4 \pm 19.1$ & 0.081 \\
\hline Gluteus muscle & $0.2 \pm 0.05$ & $0.2 \pm 0.04$ & $7.9 \pm 21.7$ & 0.100 & $0.3 \pm 0.1$ & $0.3 \pm 0.1$ & $2.7 \pm 27.9$ & 0.364 \\
\hline Liver & $4.5 \pm 0.6$ & $3.7 \pm 0.6$ & $15.7 \pm 7.3$ & $<0.001$ & $5.8 \pm 0.7$ & $4.9 \pm 0.7$ & $14.6 \pm 5.7$ & $<0.001$ \\
\hline Spleen & $3.2 \pm 0.9$ & $2.1 \pm 0.6$ & $34.3 \pm 11.1$ & $<0.001$ & $3.6 \pm 1.1$ & $2.5 \pm 0.6$ & $28.3 \pm 13.4$ & 0.001 \\
\hline Parotid glands & $9.1 \pm 2.5$ & $6.9 \pm 2.9$ & $26.1 \pm 16.9$ & 0.003 & $15.3 \pm 3.9$ & $11.6 \pm 3.8$ & $23.9 \pm 14.0$ & 0.001 \\
\hline Submandibular glands & $8.9 \pm 3.5$ & $5.8 \pm 2.9$ & $34.7 \pm 18.7$ & 0.001 & $15.1 \pm 6.2$ & $9.9 \pm 4.8$ & $35.0 \pm 11.2$ & $<0.001$ \\
\hline Lacrimal glands & $6.0 \pm 1.3$ & $3.4 \pm 1.2$ & $41.9 \pm 18.9$ & $<0.001$ & $9.9 \pm 3.4$ & $5.1 \pm 1.9$ & $48.5 \pm 12.5$ & $<0.001$ \\
\hline Colon & $5.9 \pm 1.2$ & $3.3 \pm 1.2$ & $45.1 \pm 13.8$ & $<0.001$ & $7.5 \pm 1.4$ & $4.2 \pm 1.3$ & $43.8 \pm 12.9$ & $<0.001$ \\
\hline Urinary bladder & $33.8 \pm 9.9$ & $27.9 \pm 10.2$ & $14.8 \pm 30.2$ & 0.054 & $73.0 \pm 29.3$ & $63.5 \pm 33.8$ & $14.4 \pm 32.5$ & 0.124 \\
\hline Kidneys & $17.2 \pm 4.6$ & $12.1 \pm 2.2$ & $27.4 \pm 12.1$ & 0.001 & $37.5 \pm 9.6$ & $27.4 \pm 8.0$ & $23.5 \pm 26.1$ & 0.012 \\
\hline
\end{tabular}

${ }^{*}$ Adjusted for multiple testing using Benjamini-Hochberg method.

Boldface values indicate statistical significance at $a=0.05$ level.

$P=0.11)$, gluteus muscle $(2.67 \% \pm 27.96 \%, P=0.61)$, and urinary bladder $(14.45 \% \pm 32.49 \%, P=0.19)$ did not reach statistical significance.

The most reproducible trend was seen in the parotid, submandibular, and lacrimal glands; liver; spleen; and bowel, with a lower SUL $\mathrm{S}_{\text {mean }}$ and $\mathrm{SUL}_{\max }$ on PET/CT images being found for all patients after MSG administration than for control patients. The comparison between MSG and control SUL $\mathrm{SLx}_{\text {max }}$ is shown in Figure 2 .

When the MSG amount was normalized to body weight, no significant dose-response relationship could be demonstrated for percentage changes in salivary gland and renal $\mathrm{SUL}_{\max }$
(Supplemental Fig. 1; supplemental materials are available at http://jnm.snmjournals.org).

\section{Malignant Lesions}

At least 1 lesion characteristic of PCa was detected in each patient. Active disease was most often characterized in lymph nodes $(67.6 \%)$, followed by bone $(29.6 \%)$ and prostate bed or seminal vesicles $(2.8 \%)$. Seven participants $(70 \%)$ had disease in more than 1 site. Overall, 142 lesions were detected on both MSG and control images, with a significantly higher median $\mathrm{SUL}_{\text {peak }}$ in the control group than in the MSG group, at 4.13 versus 3.01, respectively $(P<0.001)$, and a median $\mathrm{SUL}_{\text {mean }}$ of 2.88 versus 1.57 , respectively $(P<0.001)$, and a median $\mathrm{SUL}_{\max }$ of 4.36 versus 2.78 , respectively $(P<0.001)$ (Table 3). The decrease was significant for local recurrences, lymph nodes, and osseous metastases (Fig. 3). All lesions were visible on both MSG and control images, with the exception of 2 osseous metastases that were visible on control images only.

\section{Adverse Events}

Regarding vital signs for the MSG group, blood pressure changed from $156.80 \pm$ $12.03 / 92.20 \pm 8.57 \mathrm{~mm} \mathrm{Hg}$ before MSG administration to $154.90 \pm 15.63 / 84.10 \pm$ $7.63 \mathrm{~mm} \mathrm{Hg}$ immediately before the scan; the respective values were $71.70 \pm 10.67$ $\mathrm{bpm}$ to $86.70 \pm 12.31 \mathrm{bpm}$ for heart rate and $97.90 \% \pm 1.66 \%$ to $97.20 \% \pm 1.87 \%$ for pulse oximetry. In the placebo group, blood pressure changed from $153.40 \pm$ $18.66 / 90.60 \pm 6.62 \mathrm{~mm} \mathrm{Hg}$ before placebo administration to $151.80 \pm 17.54 / 85.70 \pm$ $7.21 \mathrm{~mm} \mathrm{Hg}$ at $2 \mathrm{~h}$ after radiotracer

FIGURE 2. SUL $L_{\max }$ for normal tissues in control and MSG PET/CT studies. 
TABLE 3

Comparison of ${ }^{18} \mathrm{~F}$-DCFPyl Uptake in Malignant Lesions for Control and MSG Studies

\begin{tabular}{lcccc}
\hline Parameter & Control study $(n=142)$ & MSG study $(n=140)$ & Decrease $(\%)$ & $P$ \\
\hline SUL & $4.4[1.1,30.3]$ & $2.8[0.7,23.4]$ & $32.8[-1.3,74.5]$ & $<0.001$ \\
SUL & $2.9[0.9,17.1]$ & $1.6[0.6,14.5]$ & $29.1[0.0,73.5]$ & $<0.001$ \\
SUL & $4.1[0.7,21.1]$ & $3.0[0.5,16.8]$ & $30.1[-46.6,71.6]$ & $<0.001$
\end{tabular}

All values are statistically significant at $\alpha=0.05$ level. Data are median followed by range in brackets.

injection; the respective values were $68.40 \pm 7.95 \mathrm{bpm}$ to $74.20 \pm$ $11.07 \mathrm{bpm}$ for heart rate and $98.20 \% \pm 1.75 \%$ to $97.90 \% \pm 1.52 \%$ for pulse oximetry. Except for heart rate in the MSG group, the differences in these values were not statistically significant. Heart rate alterations were considered clinically insignificant. There were no adverse events during the scans.

\section{DISCUSSION}

PSMA-targeting RLT is a promising treatment with a significant impact on PCa management (27-29). ${ }^{177} \mathrm{Lu}-$ and ${ }^{225}$ Ac-labeled PSMA ligands have shown efficacy in metastatic castration-resistant PCa patients, but physiologic tracer uptake in salivary glands and kidneys can cause xerostomia and potential for nephrotoxicity $(30,31)$. The fact that radiolabeled anti-PSMA antibodies have low uptake in these organs supports the hypothesis that beyond PSMA expression, the accumulation of small-molecule PSMA inhibitors could be partially attributed to off-target binding (32,33). Although this undesired uptake of PSMA ligands in normal PSMA-expressing organs does not hinder diagnostic interpretation, it imposes a limit on the maximum tolerable dose for PSMA-RLT. Notwithstanding the fact that xerostomia typically appears after the second or third cycle of PSMA-RLT, discontinuation of this mode of therapy has been reported in patients treated with $\alpha$-emitters (34). Patients with

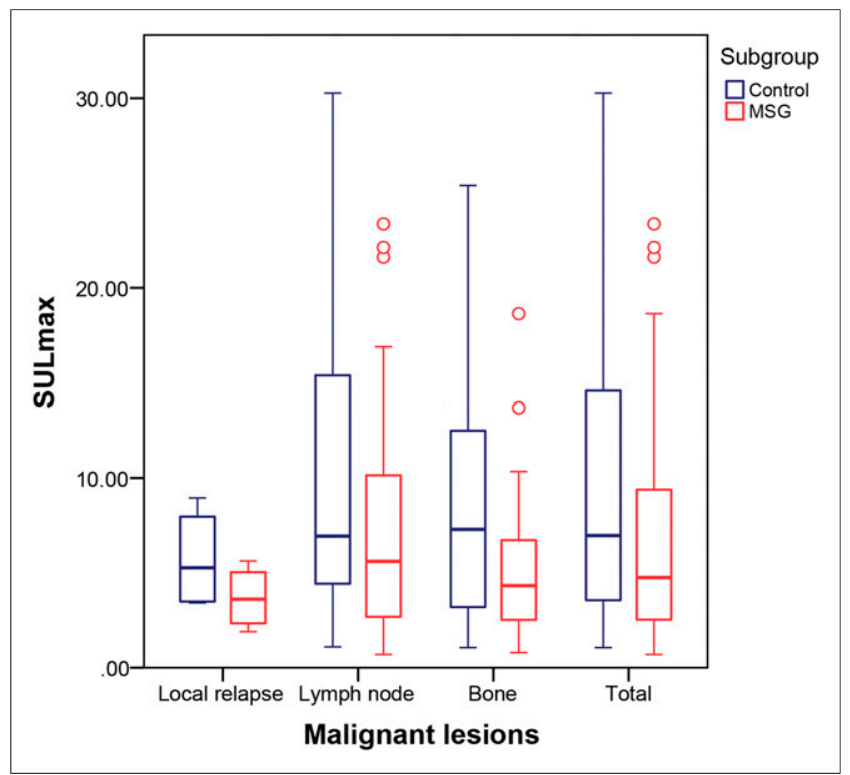

FIGURE 3. Comparison of SUL $L_{\max }$ for local relapses, lymph node metastases, osseous metastases, and total malignant lesions provided by control and MSG PET/CT studies. late-stage, heavily pretreated, resistant metastatic castration-resistant PCa constitute the main category of patients in whom these treatments have thus far been offered. The poor life expectancy of this group likely masks the emergence of late radiation-induced kidney failure, which generally requires 2 or more years to manifest (35). However, renal toxicity might become a more significant concern if PSMA-RLT were to be initiated in patients at an early stage of highrisk PCa with metastatic or oligometastatic disease (36). A protection approach to the meaningful mitigation of toxicities associated with PSMA radiotherapeutics would be useful to enable the broadest, earliest, and most effective use of these radiotherapeutics.

A few investigations have suggested protective approaches to mitigating PSMA radioligand accumulation in these organs. Rousseau et al. (24) reported that intraperitoneal injection of up to a $164 \mathrm{mg} / \mathrm{kg}$ dose of MSG in LNCap tumor-bearing NOD SCID $\gamma$ (NSG) mice resulted in lower salivary and kidney uptake of ${ }^{68} \mathrm{Ga}$-PSMA-11 in a dose-dependent manner, whereas tumor uptake was unaffected. Considering the fact that most PSMA ligands integrate a glutamate moiety to bind to PSMA, it was postulated that the administration of MSG could act by blocking nonspecific binding in healthy organs (24).

Hillier et al. showed nearly complete blockade of tumor and kidney uptake of the PSMA inhibitor ${ }^{123}$ I-MIP-1095 after coinjection of 2-PMPA (37). The potential of 2-PMPA to selectively block kidney uptake without affecting $\mathrm{LNCaP}$ tumor uptake was demonstrated by Kratochwil et al. through administration of 2-PMPA at 1 or $16 \mathrm{~h}$ after injection of the PSMA inhibitors ${ }^{99 \mathrm{~m}}$ Tc-MIP-1404 and ${ }^{125}$ I-MIP-1095, respectively (22). Chatalic et al. showed an improved tumor-to-kidney absorbed dose ratio by coinjection of 2-PMPA with ${ }^{111} \mathrm{In} /{ }^{177} \mathrm{Lu}$-PSMA I\&T, which was accompanied by a reduction in tumor uptake $(21)$.

Among recently developed orally available prodrugs of 2PMPA (23), JHU-2545 has been administered in a small number of metastatic castration-resistant PCa patients $15 \mathrm{~min}$ before injection of ${ }^{177} \mathrm{Lu}-\mathrm{PSMA}-617$ and was found to increase the metastasis or salivary gland dose ratio to $350 \%-550 \%$ of control values and the metastasis or kidney dose ratio to between $190 \%$ and $650 \%$ of control. On the basis of the available dosimetry, Nedelcovych et al. concluded that this prodrug could increase the cumulative allowable ${ }^{177} \mathrm{Lu}$-PSMA-617 dose by 2- to 6-fold (38).

Supported by favorable preclinical data obtained with ${ }^{68} \mathrm{Ga}-$ PSMA-11, the present study aimed to assess orally administered MSG as a potential means of reducing normal-organ PSMA-targeting radiotracer uptake in human subjects. We observed a significant decrease in $\mathrm{SUL}_{\max }$ on images obtained after MSG administration in the parotids, submandibular glands, kidneys, lacrimal glands, liver, spleen, and bowel. A mildly lower blood pool $\mathrm{SUL}_{\text {mean }}$ was also observed after MSG administration. Hence, our results indicate that the uptake of ${ }^{18} \mathrm{~F}-\mathrm{DCFPyL}$ in normal tissues was blocked 
by the administration of MSG, with the highest effect being noted in the lacrimal glands, followed by the colon. However, radiotracer uptake in malignant lesions was unfortunately lower on scans performed after MSG administration than on placebo scans.

The findings of this study are in line with our previously reported preclinical results, except for the decrease in malignant prostate lesions, which was not observed in tumor-bearing mice in the study conducted with ${ }^{68}$ Ga-PSMA-11. Roy et al. recently performed an ${ }^{18} \mathrm{~F}$-DCFPyL autoradiography and biodistribution study in human, mouse, rat, cynomolgus, and rhesus species and indicated that the binding affinity of ${ }^{18} \mathrm{~F}-\mathrm{DCFPyL}$ for PSMA was similar across the tested species, although the PSMA expression levels varied. The human submandibular gland exhibited approximately 2-fold lower PSMA expression than the baboon submandibular gland, whereas rodents showed the lowest PSMA levels, with mice being 10-fold higher than rats. Cynomolgus and rhesus monkeys had 2- to 3-fold lower submandibular gland PSMA levels than humans (39). Differences in PSMA expression patterns between human organs and their murine homologs, differences in relative binding affinities between ${ }^{68} \mathrm{Ga}$-PSMA- 11 and ${ }^{18} \mathrm{~F}$-DCFPyL, and differences in the pharmacokinetic properties of MSG absorption and clearance between mice and humans may contribute to the differences noted between murine and human studies.

All except 2 lesions were visible on our MSG images; the sample size was not designed to assess the impact of MSG administration on the diagnostic sensitivity for PET imaging. This study aimed at evaluating the potential for MSG to reduce activity retention in the kidneys and salivary glands, with the perspective of eventually using a similar approach for RLT. The choice of a diagnostic rather than therapeutic radiopharmaceutical was made for ethical reasons, to avoid compromising a potentially beneficial therapy with an intervention of unknown benefit.

At the oral doses we used in this study, no significant adverse events occurred after the administration of MSG. MSG is a widely studied food additive, with an excellent track record of safety (40). As an orally administered condiment, it would have been an ideal candidate compound for kidney and salivary gland protection because of its low cost, widespread availability, and low toxicity.

\section{CONCLUSION}

Our study showed that oral administration of MSG significantly reduced ${ }^{18} \mathrm{~F}$-DCFPyL uptake in salivary glands, kidneys, and other normal organs in human subjects. However, MSG also caused a corresponding decrease in tumor uptake, which limits the benefits of this approach for enhancing the therapeutic ratio of PSMARLT. Efforts to further improve our understanding of the mechanisms of PSMA radioligands in normal organs may result in more effective preventive and therapeutic strategies. Further investigations are warranted to identify compounds capable of selectively blocking both specific and nonspecific binding of PSMA radioligands in the salivary glands and kidneys to protect these organs without affecting tumor uptake.

\section{DISCLOSURE}

This study was performed with the financial support of the BC Cancer Foundation, CIHR grant FDN-148465, and the BC Leadership Chair in Functional Cancer Imaging. Dr. François Bénard is cofounder, director, and shareholder of Alpha-9 Theranostics, a radiopharmaceutical company. No other potential conflict of interest relevant to this article was reported.
KEY POINTS

QUESTION: Does orally administered MSG reduce kidney and salivary gland radiation exposure when a PSMA-targeting radiotracer is used in human subjects?

PERTINENT FINDINGS: In this analysis of a prospective, randomized, double-blind, placebo-controlled intraindividual trial, MSG significantly reduced SUL $\mathrm{Sax}_{\max }$ in the parotids $(24 \% \pm 14 \%)$, submandibular glands $(35 \% \pm 11 \%)$, and kidneys $(23 \% \pm 26 \%)$, with no adverse events.

IMPLICATIONS FOR PATIENT CARE: MSG is a candidate compound for kidney and salivary gland protection during PSMAtargeting RLT.

\section{REFERENCES}

1. Siegel RL, Miller KD, Jemal A. Cancer statistics, 2019. CA Cancer J Clin. 2019;69:7-34.

2. Sharifi N, Dahut WL, Steinberg SM, et al. A retrospective study of the time to clinical endpoints for advanced prostate cancer. BJU Int. 2005;96:985-989.

3. de Bono JS, Oudard S, Ozguroglu M, et al. Prednisone plus cabazitaxel or mitoxantrone for metastatic castration-resistant prostate cancer progressing after docetaxel treatment: a randomised open-label trial. Lancet. 2010;376:1147-1154.

4. de Bono JS, Logothetis CJ, Molina A, et al. Abiraterone and increased survival in metastatic prostate cancer. N Engl J Med. 2011;364:1995-2005.

5. Kantoff PW, Higano CS, Shore ND, et al. Sipuleucel-T immunotherapy for castration-resistant prostate cancer. N Engl J Med. 2010;363:411-422.

6. Beer TM, Armstrong AJ, Rathkopf DE, et al. Enzalutamide in metastatic prostate cancer before chemotherapy. N Engl J Med. 2014;371:424-433.

7. Parker C, Nilsson S, Heinrich D, et al. Alpha emitter radium-223 and survival in metastatic prostate cancer. N Engl J Med. 2013;369:213-223.

8. Pinto JT, Suffoletto BP, Berzin TM, et al. Prostate-specific membrane antigen: a novel folate hydrolase in human prostatic carcinoma cells. Clin Cancer Res. 1996;2: 1445-1451.

9. Maurer T, Eiber M, Schwaiger M, Gschwend JE. Current use of PSMA-PET in prostate cancer management. Nat Rev Urol. 2016;13:226-235.

10. Rahbar K, Ahmadzadehfar H, Kratochwil C, et al. German multicenter study investigating ${ }^{177} \mathrm{Lu}-\mathrm{PSMA}-617$ radioligand therapy in advanced prostate cancer patients. J Nucl Med. 2017;58:85-90.

11. Kratochwil C, Bruchertseifer F, Rathke H, et al. Targeted alpha-therapy of metastatic castration-resistant prostate cancer with ${ }^{225}$ Ac-PSMA-617: dosimetry estimate and empiric dose finding. J Nucl Med. 2017;58:1624-1631.

12. Hofman MS, Violet J, Hicks RJ, et al. [ $\left.{ }^{177} \mathrm{Lu}\right]-P S M A-617$ radionuclide treatment in patients with metastatic castration-resistant prostate cancer (LuPSMA trial): a single-centre, single-arm, phase 2 study. Lancet Oncol. 2018;19:825-833.

13. Bouchelouche K, Turkbey B, Choyke PL. PSMA PET and radionuclide therapy in prostate cancer. Semin Nucl Med. 2016;46:522-535.

14. von Eyben FE, Roviello G, Kiljunen T, et al. Third-line treatment and ${ }^{177} \mathrm{Lu}-$ PSMA radioligand therapy of metastatic castration-resistant prostate cancer: a systematic review. Eur J Nucl Med Mol Imaging. 2018;45:496-508.

15. Sarnelli A, Belli ML, Di Iorio V, et al. Dosimetry of ${ }^{177}$ Lu-PSMA-617 after mannitol infusion and glutamate tablet administration: preliminary results of EUDRACT/RSO 2016-002732-32 IRST protocol. Molecules. 2019;24:621.

16. Baum RP, Langbein T, Singh A, et al. Injection of botulinum toxin for preventing salivary gland toxicity after PSMA radioligand therapy: an empirical proof of a promising concept. Nucl Med Mol Imaging. 2018;52:80-81.

17. Rathke H, Kratochwil C, Hohenberger R, et al. Initial clinical experience performing sialendoscopy for salivary gland protection in patients undergoing ${ }^{225}$ Ac-PSMA-617 RLT. Eur J Nucl Med Mol Imaging. 2019;46:139-147.

18. Wester HJ, Schottelius M. PSMA-targeted radiopharmaceuticals for imaging and therapy. Semin Nucl Med. 2019;49:302-312.

19. Matteucci F, Mezzenga E, Caroli P, et al. Reduction of ${ }^{68} \mathrm{Ga}$-PSMA renal uptake with mannitol infusion: preliminary results. Eur J Nucl Med Mol Imaging. 2017;44:2189-2194.

20. Jackson PF, Cole DC, Slusher BS, et al. Design, synthesis, and biological activity of a potent inhibitor of the neuropeptidase $\mathrm{N}$-acetylated $\alpha$-linked acidic dipeptidase. J Med Chem. 1996;39:619-622.

21. Chatalic KL, Heskamp S, Konijnenberg M, et al. Towards personalized treatment of prostate cancer: PSMA I\&T, a promising prostate-specific membrane antigentargeted theranostic agent. Theranostics. 2016;6:849-861. 
22. Kratochwil C, Giesel FL, Leotta K, et al. PMPA for nephroprotection in PSMAtargeted radionuclide therapy of prostate cancer. J Nucl Med. 2015;56:293-298.

23. Majer P, Jančařík A, Krečmerová M, et al. Discovery of orally available prodrugs of the glutamate carboxypeptidase II (GCPII) inhibitor 2-phosphonomethylpentanedioic acid (2-PMPA). J Med Chem. 2016;59:2810-2819.

24. Rousseau E, Lau J, Kuo H-T, et al. Monosodium glutamate reduces ${ }^{68}$ Ga-PSMA11 uptake in salivary glands and kidneys in a preclinical prostate cancer model. $J$ Nucl Med. 2018;59:1865-1868.

25. Bouvet V, Wuest M, Jans H-S, et al. Automated synthesis of $\left[{ }^{18} \mathrm{~F}\right] \mathrm{DCFPyL}$ via direct radiofluorination and validation in preclinical prostate cancer models. EJNMMI Res. 2016;6:40.

26. Benjamini Y, Hochberg Y. Controlling the false discovery rate: a practical and powerful approach to multiple testing. J R Stat Soc Series B Stat Methodol. 1995;57:289-300.

27. Eiber M, Fendler WP, Rowe SP, et al. Prostate-specific membrane antigen ligands for imaging and therapy. J Nucl Med. 2017;58(suppl):67S-76S.

28. Virgolini I, Decristoforo C, Haug A, Fanti S, Uprimny C. Current status of theranostics in prostate cancer. Eur J Nucl Med Mol Imaging. 2018;45:471-495.

29. Rousseau E, Wilson D, Lacroix-Poisson F, et al. A prospective study on ${ }^{18} \mathrm{~F}$ DCFPyL PSMA PET/CT imaging in biochemical recurrence of prostate cancer. $J$ Nucl Med. 2019;60:1587-1593.

30. Afshar-Oromieh A, Babich JW, Kratochwil C, et al. The rise of PSMA ligands for diagnosis and therapy of prostate cancer. J Nucl Med. 2016;57(suppl):79S-89S.

31. Ristau BT, O'Keefe DS, Bacich DJ. The prostate-specific membrane antigen: lessons and current clinical implications from 20 years of research. Urol Oncol. 2014;32:272-279.

32. Holland JP, Divilov V, Bander NH, Smith-Jones PM, Larson SM, Lewis JS. ${ }^{89} \mathrm{Zr}-$ DFO-J591 for immunoPET of prostate-specific membrane antigen expression in vivo. J Nucl Med. 2010;51:1293-1300.
33. Silver DA, Pellicer I, Fair WR, Heston WD, Cordon-Cardo C. Prostate-specific membrane antigen expression in normal and malignant human tissues. Clin Cancer Res. 1997;3:81-85.

34. Kratochwil C, Bruchertseifer F, Rathke H, et al. Targeted $\alpha$-therapy of metastatic castration-resistant prostate cancer with ${ }^{225}$ Ac-PSMA-617: swimmer-plot analysis suggests efficacy regarding duration of tumor control. J Nucl Med. 2018;59: 795-802.

35. Valkema R, Pauwels SA, Kvols LK, Kwekkeboom DJ. Long-term follow-up of renal function after peptide receptor radiation therapy with ${ }^{90} \mathrm{Y}^{-D O T A}{ }^{0}, \mathrm{Tyr}^{3}$ octreotide and ${ }^{177} \mathrm{Lu}^{-D O T A}{ }^{0}, \mathrm{Tyr}^{3}$-octreotate [abstract]. J Nucl Med. 2005; 46(suppl):83S

36. von Eyben FE, Baumann GS, Baum RP. PSMA diagnostics and treatments of prostate cancer become mature. Clin Transl Imaging. 2018;6:145-148.

37. Hillier SM, Maresca KP, Femia FJ, et al. Preclinical evaluation of novel glutamate-urea-lysine analogues that target prostate-specific membrane antigen as molecular imaging pharmaceuticals for prostate cancer. Cancer Res. 2009;69: 6932-6940.

38. Nedelcovych MT, Dash RP, Wu Y, et al. JHU-2545 selectively shields salivary glands and kidneys during PSMA-targeted radiotherapy. bioRxiv website. https:// www.biorxiv.org/content/10.1101/457085v1.full. Published October 30, 2020. Accessed July 23, 2020.

39. Roy J, Warner BM, Basuli F, et al. Comparison of prostate-specific membrane antigen expression levels in human salivary glands to non-human primates and rodents. Cancer Biother Radiopharm. 2020;35:284-291.

40. Mortensen A, Aguilar F, Crebelli R, et al. Re-evaluation of glutamic acid (E 620 ), sodium glutamate (E 621), potassium glutamate (E 622), calcium glutamate (E 623), ammonium glutamate (E 624) and magnesium glutamate (E 625) as food additives. EFSA J. 2017;15:e04910. 